\title{
Communication \\ A Point-of-Care Assay Based on Reflective Phantom Interface (RPI) Technology for Fast, Multi-Toxin Screening in Wheat
}

\author{
Matteo Salina ${ }^{1}$, Giovanni Tagliabue ${ }^{1}\left(\mathbb{D}\right.$, Roberta Ghizzoni ${ }^{2}$, Valeria Terzi $\left.{ }^{2}{ }^{(}\right)$and Caterina Morcia ${ }^{2, *(1)}$ \\ 1 Proxentia, Viale Ortles 22/4, I-20139 Milano, Italy; matteo.salina@proxentia.com (M.S.); \\ giovanni.tagliabue@proxentia.com (G.T.) \\ 2 Consiglio Per la Ricerca in Agricoltura e L'analisi Dell'economia Agraria-Centro di Ricerca Genomica e \\ Bioinformatica (CREA-GB), Via San Protaso 302, I-29017 Fiorenzuola d'Arda, Italy; \\ roberta.ghizzoni@crea.gov.it (R.G.); valeria.terzi@crea.gov.it(V.T.) \\ * Correspondence: caterina.morcia@crea.gov.it; Tel.: +39-0523-98-3758
}

check for

updates

Citation: Salina, M.; Tagliabue, G.; Ghizzoni, R.; Terzi, V.; Morcia, C. A Point-of-Care Assay Based on Reflective Phantom Interface (RPI) Technology for Fast, Multi-Toxin Screening in Wheat. Agronomy 2022, 12, 493. https://doi.org/10.3390/ agronomy12020493

Academic Editors: Colm Carraher and Mario Cunha

Received: 26 December 2021 Accepted: 14 February 2022 Published: 16 February 2022

Publisher's Note: MDPI stays neutral with regard to jurisdictional claims in published maps and institutional affiliations.

Copyright: (C) 2022 by the authors. Licensee MDPI, Basel, Switzerland. This article is an open access article distributed under the terms and conditions of the Creative Commons Attribution (CC BY) license (https:// creativecommons.org/licenses/by/ $4.0 /)$.

\begin{abstract}
Mycotoxigenic fungi can colonize small grain cereals causing severe yield losses and grain contaminations. Fusaria can be responsible for the contamination of wheat grains and derived products via several classes of mycotoxins, negatively impacting human and animal health. Among the strategies to control mycotoxins are analytical tools for their identification and quantification from field to food and feed. A fast multi-toxin assay based on reflective phantom interface (RPI) technology was developed to identify and quantify deoxynivalenol, zearalenone, and T-2/HT-2 toxins. The PRX analytical workflow was organized as follows: a fast mycotoxins extraction step followed by an analytical step carried out in a system composed of three elements: (I) a universal reader able to read a series of (II) cartridges that incorporate the RPI technology and (III) a software that analyzes data and gives feedback on the results. The assay was evaluated in wheat reference samples at known levels of toxin contaminations and on naturally contaminated grain samples. The results obtained suggest that the assay can be considered a useful screening tool for point-of-care and point-of-sale control of toxins contamination along wheat production and transformation chains.
\end{abstract}

Keywords: mycotoxins; deoxynivalenol; zearalenone; T-2/HT-2; reflective phantom interface; small grain cereals; point-of-care test; biosensor

\section{Introduction}

Agri-food production's health can be jeopardized by several contaminants, such as heavy metals, pesticides, packaging residues, or undesired by-products formed during a transformation phase. The pathogenic microbiological component plays a key role as it can lead to reductions in field production and decrease in quality of derived products. A relevant associated issue is mycotoxin contamination. The FAO (Food and Agriculture Organization of the United Nations) estimates mycotoxin occurrence above EU (European Union) and Codex limits in $25 \%$ of raw materials and food/feed, but Eskola et al. [1] underlined that $60-80 \%$ of worldwide production is contaminated above the detectable levels. Mycotoxins are secondary metabolites produced by different types of fungi, including Aspergillus, Penicillium, Alternaria, and Fusarium. Toxins can enter the food chain via contaminated crops intended for food and feed production; they represent a high risk for human and animal health due to their toxic effects, including genotoxicity, carcinogenicity, immunotoxicity, mutagenicity, nephrotoxicity, and teratogenicity [2]. Microbiological safety of raw materials is, therefore, fundamental to improve the final product quality. Mycotoxin contamination control is of great relevance for small grain cereals as they fulfill a primary role in worldwide human nutrition with high per capita consumption and wide utilization, estimated at 2700 million tons in 2020 [3]. A primary goal in the cereals sector, intended for both human and zootechnical nutrition, is, therefore, the control and reduction of the 
risk factor determined by the presence of mycotoxins. Analytical methods for mycotoxin determination are primary tools for risk assessment and control.

The development of analytical methods for mycotoxin determination is annually reviewed [4] to discuss most recent developments and trends, together with limitations and perspectives. A methods category of increasing interest is the rapid diagnostic, mostly based on biosensors for multiplex, single or family mycotoxins detection. In particular, "point-of-care testing" (POCT) can revolutionize food chain control operations as some food characteristics can potentially be tested directly during production and/or in storage areas, therefore saving time and reducing costs. POCT devices can be designed to meet the needs of both structured laboratories and small manufacturers to identify mycotoxins immediately in the working area. The use of biosensors based on optical, piezoelectric, electrochemical transducers has been recently proposed for on-site testing of samples, as reviewed by $[4,5]$. The advantages of such biosensors range from the reduced analysis time to the high sensitivity and low costs, whereas the limitations are mainly related to the complex construction of the devices, expensive labeling, and low robustness of the assays [5]. The aim of this study was to overcome some of these limitations by developing a tool able to quantify target molecules (e.g., toxins) at reduced analytical time and cost, applicable outside specialized laboratories as portable device, and finally of simple construction.

To reach this goal, a label-free detection method can be advantageous. Accordingly, we focused on a novel optical label-free detection method called reflective phantom interface (RPI).

In this study, a POCT approach for multiplex mycotoxin detection in wheat has been developed and evaluated both in reference materials and grain samples in comparison with ELISA tests. The assay is based on a simplified extraction procedure coupled with a multi-spot immunoassay and RPI based detection technology. The test can analyze mycotoxins, namely deoxynivalenol, zearalenone, and T-2/HT-2, in cereal samples.

\section{Materials and Methods}

\subsection{Samples}

The following reference samples were used: Romer wheat QCM2W1 (Romer Labs, Tulln, Austria) and FAPAS T22146QC (Fera Science Ltd., Sand Hutton, York, UK).

A panel of grain samples with the listed characteristics was used, derived from naturally contaminated wheat plants cultivated in 2018-2019 growing season in the experimental fields of the Research Centre for Genomics and Bioinformatics and free from any fungicide treatment. The grains were milled using an analytical mill (IKA Universal mill M20, IKA-Werke $\mathrm{GmbH}$, Staufen, Germany) and stored at $4{ }^{\circ} \mathrm{C}$ until analysis.

\subsection{ELISA Assays}

The DON (deoxynivalenol) amount was determined using the kit RIDASCREEN ${ }^{\circledR}$ DON (Product code R5906, R-Biopharm AG, Darmstadt, Germany). The assay was carried out accordingly to the manufacturer's instructions. The photometric readings were carried out at $450 \mathrm{~nm}$ with a ChroMate Model 4300 microplate reader (Awareness Technology INC. Palm City, FL, USA) and the toxin concentrations were calculated using the RIDA ${ }^{\circledR}$ SOFT Win. net software (code Z9996, R-Biopharm AG, Darmstadt, Germany).

T-2/HT-2 content (as sum of mycotoxins) was determined using the Veratox for T-2/HT-2 kit (product code 8230, Neogen Corporation, Lansing, MI, USA). The assay was conducted according to the manufacturer's instructions. The photometric reading was carried out at $630 \mathrm{~nm}$ with a ChroMate Model 4300 microplate reader (Awareness Technology INC., Palm City, FL, USA). 


\subsection{PRX Test}

The PRX test was based on a multi-spot immunoassay exploiting an optical label-free detection based on Reflective Phantom Interface (RPI) [6]. The detection system is patented by ProXentia as a "Method for the direct measure of molecular interactions by detection of light reflected from multilayered functionalized dielectrics", Patent number: EP3814752. The multi-spot immunoassay was hosted on a chip. Receptors (the spots) were deposited on the internal surfaces of the cartridges. They were functionalized to bind the mycotoxins DON, ZON (zearalenone) and T-2/HT-2 by a stable covalent bond [7]. The chip was included in a cartridge.

The overall system view is reported in Figures 1 and 2. Figure 1 shows the workflow from mycotoxins extraction to the sample loading onto the cartridge, whereas Figure 2 gives a general view of the analytical system.

Sample preparation
Weigh $5 \mathrm{~g}$ of the sample, add $25 \mathrm{ml}$ of the
Extract Solution and shake for 20 seconds
Let settle the sample for 4 minutes, the sample
does not need to be clear

Figure 1. The analytical workflow for the mycotoxin detection in wheat. The procedure can be summarized in three simple steps. The figure is based on the authors' photographs.
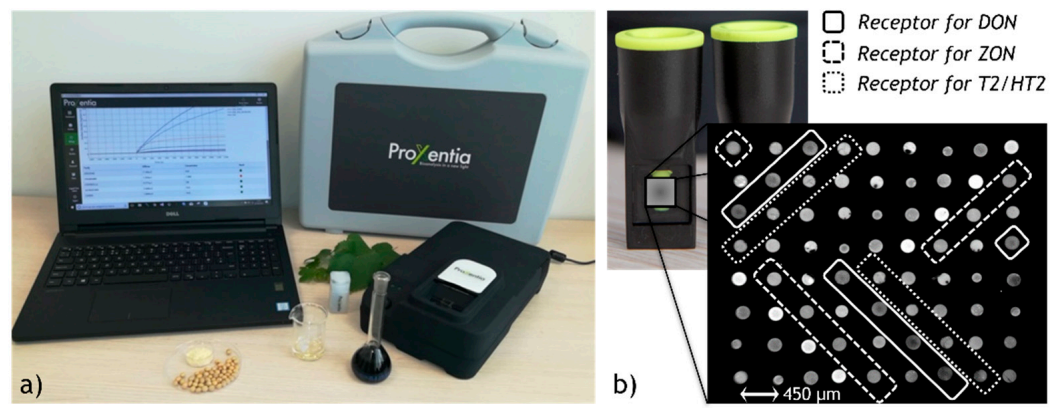

Figure 2. The system overview: (a) The PRX system was composed of three elements: (I) a universal reader able to read a series of (II) cartridges that incorporate the RPI technology, and (III) a software that analyzes the data and returns the results of the analysis to the user. (b) The cartridges and their surfaces for mycotoxins analysis in wheat. Receptors for mycotoxins are highlighted, whereas unmarked receptors are calibrators plus negative and positive controls. The figure is based on author's photographs. 
Figures 3 and 4 show key features of the DON, ZON and T-2/HT-2 receptors used in PRX assay. In particular, Figure 3 shows the kinetic parameters and specificity of the monoclonal antibodies, whereas Figure 4 shows antibodies calibration curves. Each antibody has a very high specificity and almost zero cross reactivity.
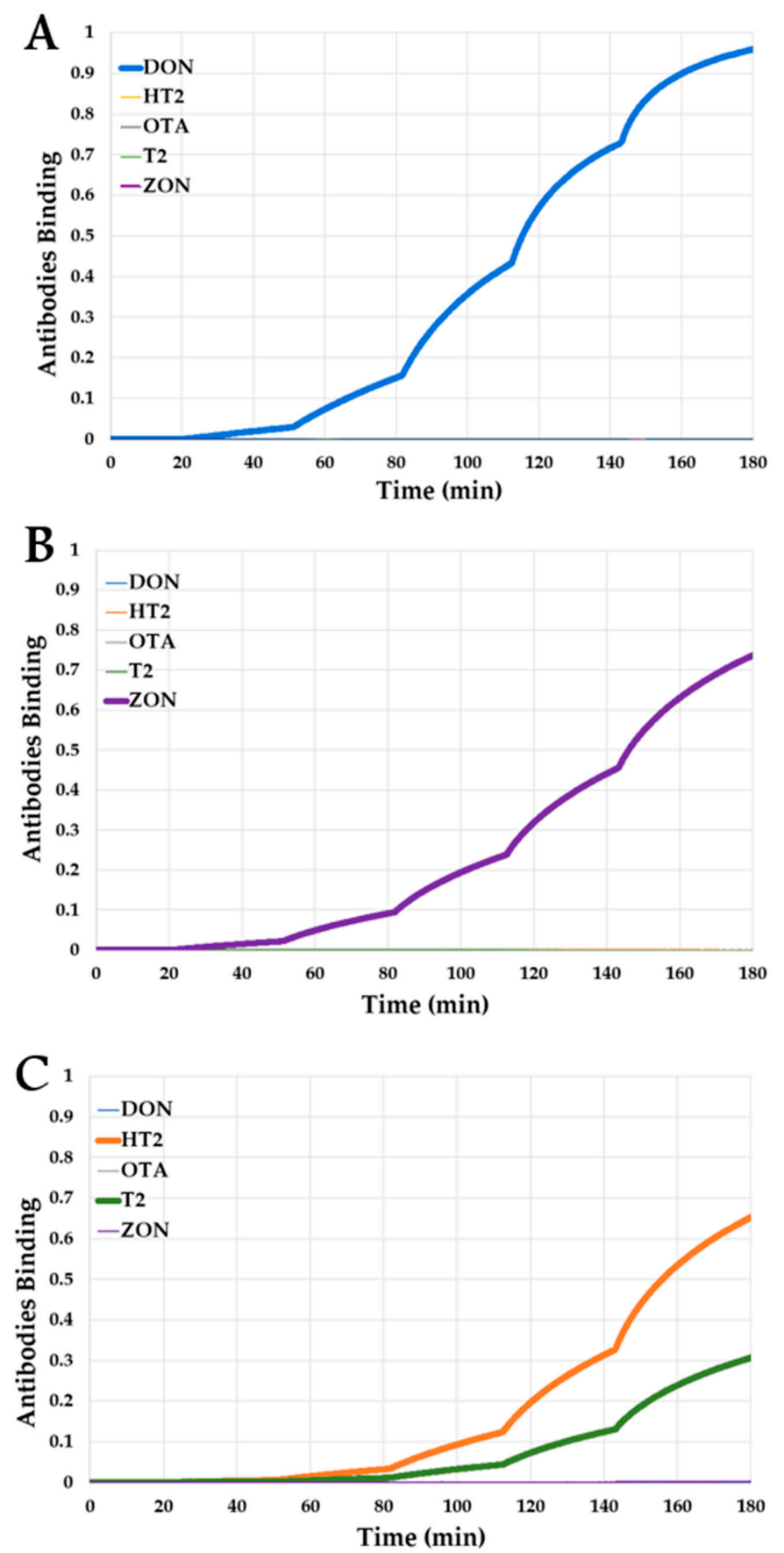

Figure 3. Characterization assay for the determination of kinetic parameters and cross-reactivity of monoclonal antibodies anti-deoxynivalenol (A), anti-zearalenone (B), and anti-T-2/HT-2 toxins (C). 

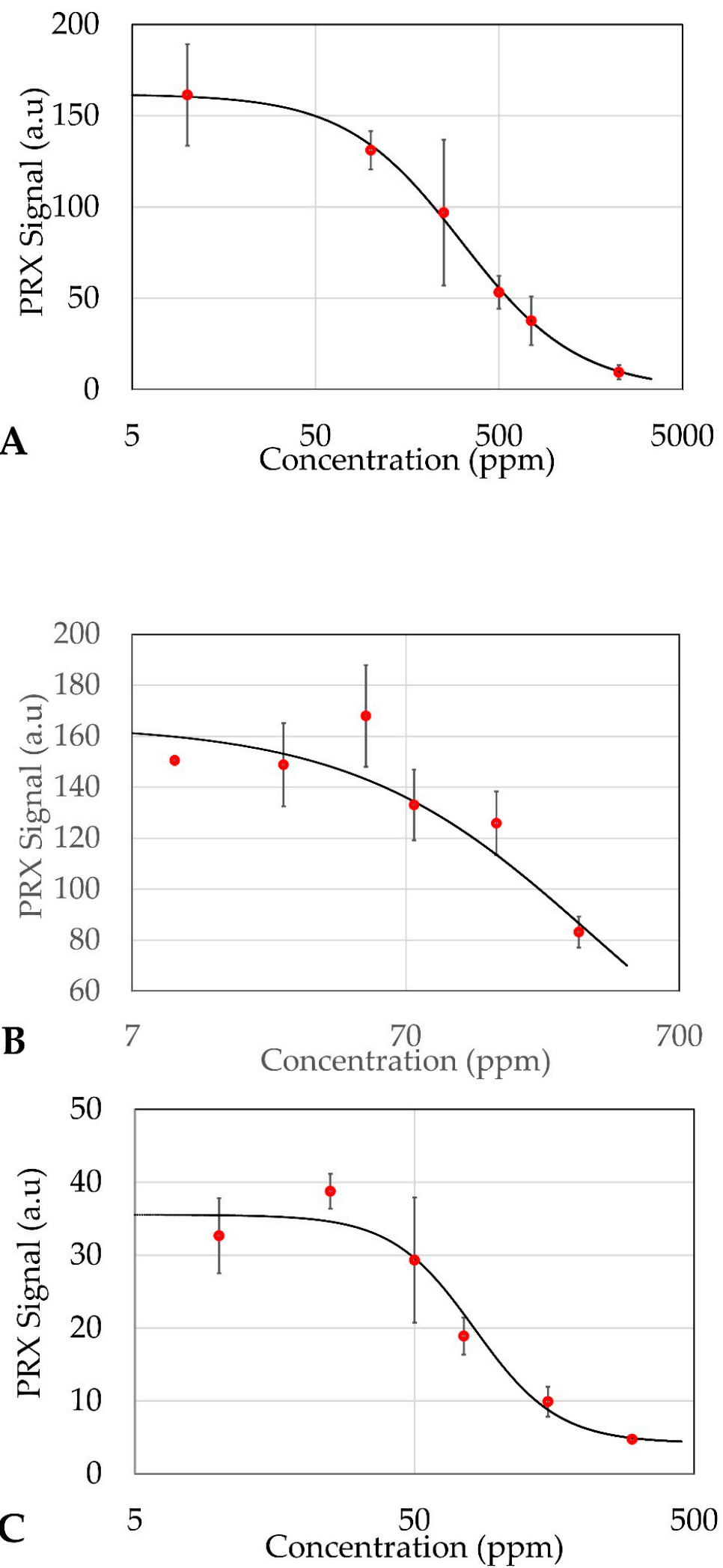

Figure 4. Calibration curves for the antibodies anti-deoxynivalenol (A), anti-T-2/HT-2 toxins (B), and anti-zearalenone $(\mathbf{C})$. The semi-log graphs show the dose-response curve made with artificially contaminated samples prepared from an uncontaminated wheat flour spiked with determined amounts of mycotoxin standards. Error bars for each calibration point are reported. 
Each antibody was tested individually. As per Figure 2, the internal surface of the PRX cartridge was functionalized with various receptors (the spots) with covalent-bound mycotoxins (DON, ZON, T-2/HT-2, and OTA as negative control). The assay was carried out adding, at fixed times, increasing concentrations of antibody inside the cartridge. The graphs show the antibody binding for each receptor (expressed as normalized values against extrapolated maximum binding) versus time. The extrapolated maximum binding was calculated on the horizontal asymptote of the curve at maximum concentration. This value was assumed as the maximum binding capacity. Total antibody concentrations in the cartridge for the shown tests are: Anti-deoxynivalenol $(\mu \mathrm{g} / \mathrm{mL}): 0.02 ; 0.12 ; 0.62 ; 3.00 ; 14.43$; Anti-zearalenone ( $\mu \mathrm{g} / \mathrm{mL}): 0.02 ; 0.12 ; 0.61 ; 2.97 ; 14.40$; Anti-T-2/HT-2 toxins $(\mu \mathrm{g} / \mathrm{mL}): 0.02$; $0.13 ; 0.64 ; 3.12 ; 15.12$.

\section{Results and Discussion}

The reflective phantom Interface (RPI) patented technology harnesses very low reflectivity surfaces' properties. The bound of the target molecules modulated the reflected light through an interface with a very low reflectivity in aqueous solutions. The interaction between the targets and the receptors spotted on the surface induced an increase in the reflected light intensity that provided the readout of the assay without any kind of secondary labelling [6]. As previously reported, the analysis was carried out by a simple device formed by a compact reader, called PRX200, a disposable cartridge and a specific software. In this multi-toxins PRX assay, receptors for DON, ZON, and T-2/HT-2 were spotted on the surface of the PRX sensor that, in contact with aqueous solutions, became almost completely transparent. If a sample was contaminated, mycotoxin molecules bound to specific receptors inducing variations in reflected light. Such variations provides not only the identification of the mycotoxin present in the sample but even its quantification. As three kinds of receptors were hosted in the sensor, the simultaneous identification and quantification of DON, ZON and T-2/HT-2 was possible. Quantification was obtained referring to calibration curves built using serial dilutions of DON (ranging from 75 to $2250 \mathrm{ppb}$ ), ZON (from 25 to $300 \mathrm{ppb}$ ) and T-2/HT-2 (from 25 to $300 \mathrm{ppb}$ ).

The multi-toxins PRX assay developed for quantification of DON, ZON and T-2/HT2 was evaluated on wheat reference samples with known DON, ZON, and T-2/HT-2 contamination level (Table 1). The mycotoxins contents in reference samples, measured with the PRX assay, fell within the specified values from the supplier for all the three classes of mycotoxins, with the exception of DON concentration in Romer reference sample. The PRX assay applied to such reference sample gives, in several replicates, a lower DON value in comparison with that declared. On the contrary, the Fapas reference sample was correctly read by the assay. In both reference samples, the DON concentrations declared by the producers and detected with PRX assay were under the fixed EU and other countries limits for DON contamination in wheat. PRX assay correctly classified the Romer and Fapas reference samples under the limits fixed for DON.

Table 1. Wheat reference samples are reported, with DON, ZON, and T-2/HT-2 content values in $\mathrm{ppb}$ declared by producers and experimentally determined according to PRX assay.

\begin{tabular}{ccc}
\hline Reference Sample Code & $\begin{array}{c}\text { Mycotoxin Content Range } \\
\text { (ppb) According to Producer }\end{array}$ & $\begin{array}{c}\text { Mean Mycotoxin Content } \\
\text { (ppb) According to } \\
\text { PRX Analysis }\end{array}$ \\
\hline Fapas T22146QC & $520-1037 \mathrm{DON}$ & $602 \pm 221 \mathrm{DON}$ \\
Romer QCM2W1 & $49.1-126.2 \mathrm{ZON}$ & $84.9 \pm 22.5 \mathrm{ZON}$ \\
$31.8-81.7 \mathrm{~T}-2 / \mathrm{HT}-2$ & $81.9 \pm 0.21 \mathrm{~T}-2 / \mathrm{HT}-2$ \\
& $848-968 \mathrm{DON}$ & $457 \pm 220 \mathrm{DON}$ \\
\hline
\end{tabular}

PRX assay was evaluated on 24 grain samples harvested in the field of CREA-GB and exposed to natural Fusarium populations. ELISA tests were applied to these same field samples. DON contaminated all the field samples selected, T-2/HT-2 toxins were found 
in a subset of such samples, whereas $\mathrm{ZON}$ is not detectable in all the samples. This is not surprising because of the known occurrence of antagonisms among different fungal species. The correlation among PRX and ELISA values for DON and T-2/HT-2 was determined in the contaminated samples only. Figure 5 shows the DON quantifications obtained in grain samples with PRX approach in comparison with ELISA. A Pearsons' $r$ of 0.89 was found between PRX and ELISA based quantification.

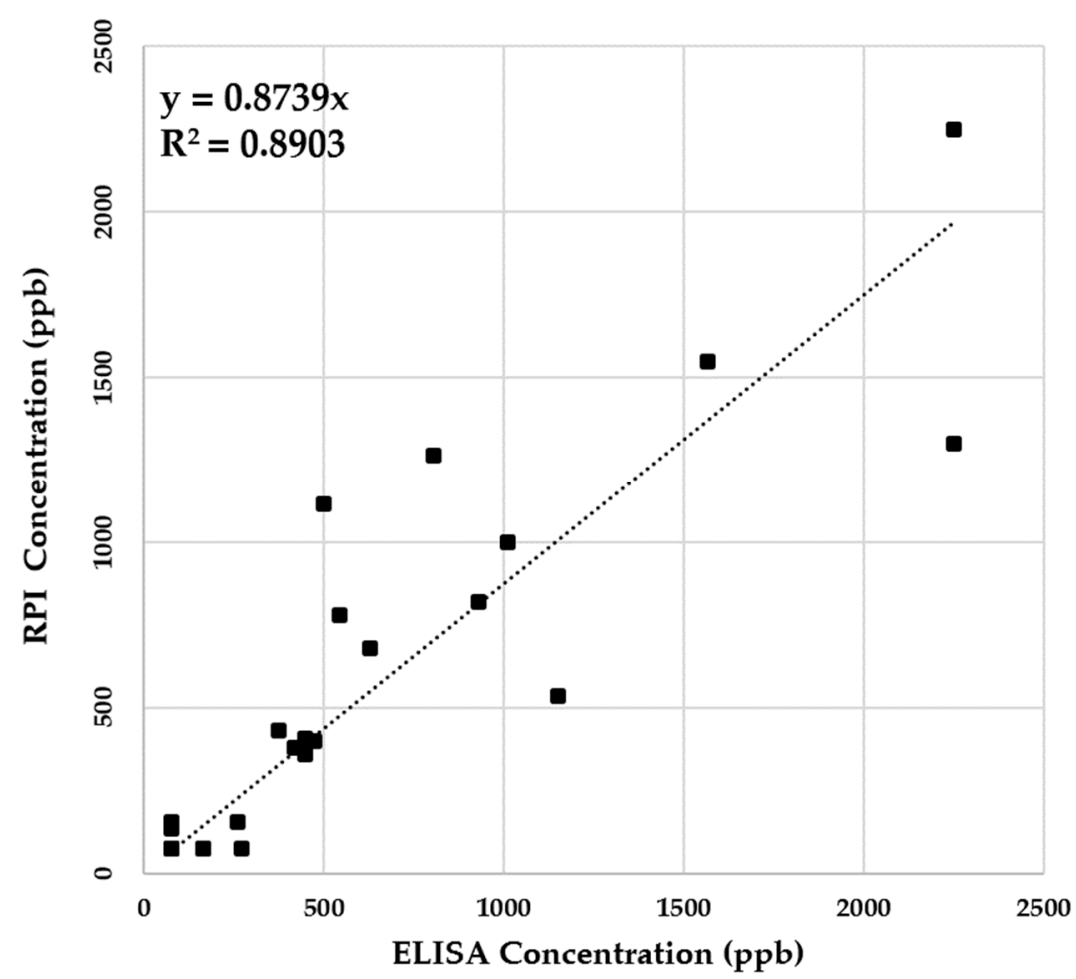

Figure 5. DON quantification (expressed in ppb) in 24 field grain samples with the two different screening approaches, ELISA test and PRX assay.

PRX assay was applied to nine grain samples whose T-2/HT-2 content was also determined with ELISA test. The data obtained are reported in Figure 6. A Pearsons' $r$ of 0.85 was found between ELISA and PRX quantification data. The PRX data on T2/HT-2 are consistent with the Fapas reference sample (31.8-81.7 ppb range) and with the ELISA data of field samples with contamination around the recommended limit of $100 \mathrm{ppb}$. Uncertainty increased in the less contaminated samples; however, our comparison was performed between two technologies and it can be hypothesized that in such lowcontaminated samples the uncertainty of the two methods can interact and impact on the final correlation value.

From the results obtained, multi-toxin PRX assay can correctly identify and quantify the three toxin classes in reference samples and, in field samples, showed high degree of correlation with ELISA data.

According to CE N. 1126/2007, 28 September 2007 [8], the DON limit in unprocessed cereals is fixed at 1250, in grain and flour for human consumption is fixed at $750 \mathrm{ppb}$ and in bread and bakery products at $500 \mathrm{ppb}$. ZON limits range from $100 \mathrm{ppb}$ in unprocessed grains to $50 \mathrm{ppb}$ in food, whereas for T-2/HT-2 the 2013/165/UE recommendation [9] is to monitor samples exceeding the limits of $100 \mathrm{ppb}$ in unprocessed grains to $25 \mathrm{ppb}$ in food. The method's sensitivity is coherent with such legal or recommended limits fixed by EU commission for these mycotoxins. 


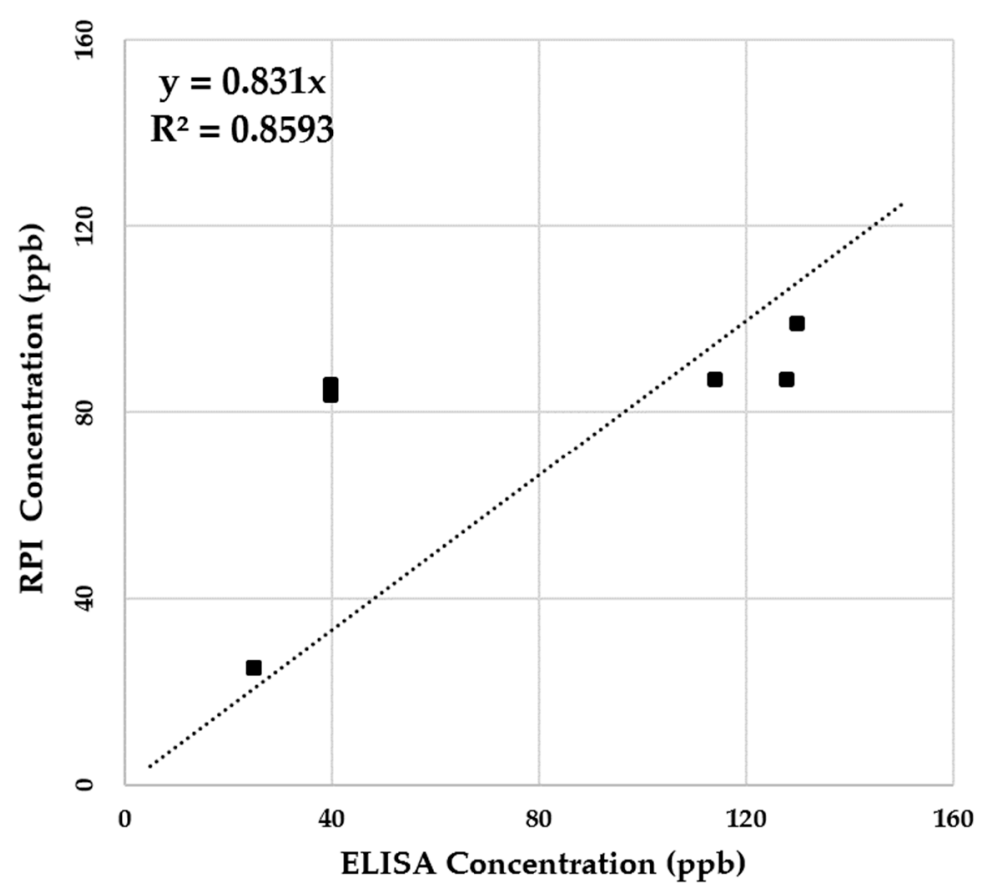

Figure 6. T-2/HT-2 quantification (expressed in $\mathrm{ppb}$ ) in 9 field grain samples with the two different screening approaches, i.e., ELISA test and PRX assay.

The method is applicable outside laboratories, in the most effective time and place to control possible contamination, thanks to the simplified mycotoxins extraction and analysis. The instrument is portable and therefore suitable for use in various situations. It should be emphasized that it is an electronic device developed for indoor use and should be kept away from heat and water sources. The analysis kit has been developed and tested for room temperature and 30-60\% relative humidity.

Several POC methods are based on capture and analysis of images through smartphones and dedicated applications [10]. On the contrary, the PRX device has an internal camera able to capture the image and a software for data processing. The choice of such a dedicated device instead of smartphone avoids developing, and validates several applications suitable for different smartphones, ensuring greater standardization of results. To ensure further data management, PRX devices can be connected via wi-fi to a network.

Multiparameter point-of-care assays are changing the face of diagnostics, allowing the detection of several analytes from a small volume of test material. Such tests are excellent in situations where it is necessary to quickly retrieve results. Paper-based and chip-based devices are the most common in the agri-food sector, but several alternatives have been proposed, based on thread, tube, cuvette, disc, glass, and nanomaterials [11]. In comparison with other point-of-care approaches, the PRX methods has some peculiarities. The method uses antibodies instead of aptamers because of their high stability and great affinity [12]. PRX method uses a cartridge hosting the antibodies. As several antibodies can be hosted in the cartridge, multi-target assays can be carried out, which is one of the major requests from the market [13]. The multiplex analysis can be tailored for specific crop contaminants, such as, importantly, wheat contaminant mycotoxins. This is another peculiarity of PRX assay: to the best of our knowledge, no other POC device is available for targeting, in multiplex, the most important mycotoxins that can contaminate small grain cereals. Another key aspect of the tool is the low analytical cost, due to three main features of the assay: the very simple and fast sample preparation ( $5 \mathrm{~min}$ ), the simplicity in conducting the analysis which can therefore be entrusted to unskilled personnel, and the possibility to organize multi-target assays. Indicatively, the cost for a multi-toxin PRX analysis is comparable with that for a single toxin with a lateral flow device. A further strength of the technology presented is that it can produce not only a qualitative-, but also a semi-quantitative analytical response. 
This is a fundamental practical aspect that allows to make fast decisions on the batch under analysis. Different interventions can be decided for batches that are within or above the limits set or recommended for different mycotoxins. It is therefore possible to introduce PRX assay during wheat harvesting, storage, milling, and subsequent transformation steps. The assay, positively evaluated on reference and real-world wheat samples, deserves further validation step expanding the number of analytical samples and evaluating its competitiveness respect to other approaches in practical applications.

\section{Conclusions}

The development of user friendly, fast, and inexpensive assays for mycotoxin detection is an actively growing research area [4,11,14-16]. Several approaches are based on colorimetric strategies for mycotoxins detection [12]. Others have found alternative routes ranging from barometric changes coupled to competitive immunoassays [17] to a latex microsphere immunochromatography platform coupled with s user-friendly Android application to analyze, report, and share the detection results [18]. There are also optical biosensors, operating variation in optical signals generated by transducer after recognition of the target molecule, which excel in simplicity, speed of detection, sensitivity, and visualization $[19,20]$. The innovation proposed by the PRX method relies on the employment of an optical label-free detection technology (RPI) in which interactions between the targets and the receptors spotted on the surface induce an increase in reflected light intensity that provides the readout of the assay without any kind of secondary labelling. The usefulness of this technique has been exemplified in a multi-toxin test for contamination control in wheat.

\section{Patents}

System for the rapid analysis of samples and corresponding reader, cartridge and method (https:/ / worldwide.espacenet.com/publicationDetails / biblio?II=0\&ND=3\&adjacent=true\& locale $=$ en_EP\&FT $=\mathrm{D} \&$ date $=20210505 \& C C=E P \& N R=3814752 \mathrm{~A} 1 \& \mathrm{KC}=\mathrm{A} 1)($ accessed on 10 February 2022).

Author Contributions: Conceptualization, M.S. and V.T.; methodology, G.T. and M.S.; software, G.T. and M.S.; investigation, R.G. and C.M.; data curation, G.T.; writing—original draft preparation, V.T. and G.T.; writing-review and editing, M.S. and C.M.; funding acquisition, V.T. and M.S. All authors have read and agreed to the published version of the manuscript.

Funding: This research was funded by European Commission, by Horizon 2020 program grant number 718855 SME instrument and by INVITE.

Data Availability Statement: Not applicable.

Acknowledgments: The support in manuscript revision given by Ilaria Carrara is acknowledged.

Conflicts of Interest: M.S. and G.T. are ProXentia employers.

\section{References}

1. Eskola, M.; Kos, G.; Elliott, C.T.; Hajšlová, J.; Mayar, S.; Krska, R. Worldwide contamination of food-crops with mycotoxins: Validity of the widely cited 'FAO estimate' of 25\%. Crit. Rev. Food Sci. Nutr. 2020, 60, 2773-2789. [CrossRef] [PubMed]

2. European Food Safety Authority-EFSA Panel on Contaminants in the Food Chain (CONTAM). Scientific Opinion on the risks for animal and public health related to the presence of T-2 and HT-2 toxin in food and feed. EFSA J. 2011, 9, 2481. [CrossRef]

3. World Food Situation. Available online: http://www.fao.org/worldfoodsituation/csdb/en (accessed on 15 November 2021).

4. Tittlemier, S.; Brunkhorst, J.; Cramer, B.; DeRosa, M.; Lattanzio, V.; Malone, R.; Maragos, C.; Stranska, M.; Sumarah, M. Developments in mycotoxin analysis: An update for 2019-2020. World Mycotoxin J. 2021, 14, 3-26. [CrossRef]

5. Agriopoulou, S.; Stamatelopoulou, E.; Varzakas, T. Advances in Analysis and Detection of Major Mycotoxins in Foods. Foods 2020, 9, 518. [CrossRef] [PubMed]

6. Tagliabue, G.; Faoro, V.; Rizzo, S.; Sblattero, D.; Saccani, A.; Riccio, G.; Bellini, T.; Salina, M.; Buscaglia, M.; Marcello, A. A label-free immunoassay for Flavivirus detection by the Reflective Phantom Interface tehnology. Biochem. Biophys. Res. Commun. 2017, 492, 558-564. [CrossRef] 
7. Method for the Direct Measure of Molecular Interactions by Detection of Light Reflected from Multilayered Functionalized Dielectrics. Patent Number: 893288 and 20120244554 . Available online: https://patents.justia.com/patent/8932880 (accessed on 16 November 2021).

8. CE N. 1126/2007. 28 September 2007. Available online: https:/ / eur-lex.europa.eu/legal-content/IT/TXT/?uri=CELEX:32007R1 126 (accessed on 16 November 2021).

9. 2013/165/UE Recommendation. Available online: https://eur-lex.europa.eu/legal-content/EN/ALL/?uri=CELEX:32013H016 5R(01) (accessed on 16 November 2021).

10. Zhang, L.; Zhang, Z.; Tian, Y.; Cui, M.; Huang, B.; Luo, T.; Zhang, S.; Wang, H. Rapid, simultaneous detection of mycotoxins with smartphone recognition-based immune microspheres. Anal. Bioanal. Chem. 2021, 13, 3683-3693. [CrossRef] [PubMed]

11. Choi, J.R.; Yong, K.W.; Choi, J.Y.; Cowie, A.C. Emerging Point-of-care Technologies for Food Safety Analysis. Sensors 2019, $19,817$. [CrossRef] [PubMed]

12. Majdinasab, M.; Ben Aissa, S.; Marty, J.L. Advances in Colorimetric Strategies for Mycotoxins Detection: Toward Rapid Industrial Monitoring. Toxins 2021, 13, 13. [CrossRef] [PubMed]

13. Nolan, P.; Auer, S.; Spehar, A.; Elliott, C.T.; Campbell, K. Current trends in rapid tests for mycotoxins. Food Addit. Contam. Part A Chem. Anal. Control Expo. Risk Assess. 2019, 36, 800-814. [CrossRef] [PubMed]

14. Jia, M.; Liao, X.; Fang, L.; Jia, B.; Liu, M.; Li, D.; Zhou, L.; Kong, W. Recent advances on immunosensors for mycotoxins in foods and other commodities. TrAC Trends Anal. Chem. 2021, 136, 116193. [CrossRef]

15. Singh, J.; Mehta, A. Rapid and sensitive detection of mycotoxins by advanced and emerging analytical methods: A review. Food Sci. Nutr. 2020, 8, 2183-2204. [CrossRef] [PubMed]

16. Mahmoudpour, M.; Ezzati Nazhad Dolatabadi, J.; Torbati, M.; Pirpour Tazehkand, A.; Homayouni-Rad, A.; de la Guardia, M. Nanomaterials and new biorecognition molecules based surface plasmon resonance biosensors for mycotoxin detection. Biosens. Bioelectron. 2019, 143, 111603. [CrossRef] [PubMed]

17. Zhang, W.; Wu, W.; Cai, C.; Hu, X.; Li, H.; Bai, Y.; Zhang, Z.; Li, P. A Sensitive, Point-of-Care Detection of Small Molecules Based on a Portable Barometer: Aflatoxins in Agricultural Products. Toxins 2020, 12, 158. [CrossRef] [PubMed]

18. Li, X.; Wang, J.; Yi, C.; Jiang, L.; Wu, J.; Chen, X.; Shen, X.; Sun, Y.; Lei, H. A smartphone-based quantitative detection device integrated with latex microsphere immunochromatography for on-site detection of zearalenone in cereals and feed. Sens. Actuators B Chem. 2019, 290, 170-179. [CrossRef]

19. Chen, H.; Zhang, L.; Hu, Y.; Zhou, C.; Lan, W.; Fu, H.; Shen, Y. Nanomaterials as optical sensors for application in rapid detection of food contaminants, quality and authenticity. Sens. Actuators B Chem. 2021, 329, 129135. [CrossRef]

20. Li, R.; Wen, Y.; Wang, F.; He, P. Recent advances in immunoassays and biosensors for mycotoxins detection in feedstuffs and foods. J. Anim. Sci. Biotechnol. 2021, 12, 108. [CrossRef] 